\title{
Sweet Dreams or Bitter Nightmare: Can We Trust on Fructose?
}

Sergio Polakof*, Jean-Louis Sébedio and Blandine Comte

INRA, UMR 1019, UNH, CRNH Auvergne, F-63000 Clermont-ferrand, France

\begin{abstract}
Fructose consumption has increased dramatically in the last century in the Western diets, associated with a concomitant development of some physiopathologies like the metabolic syndrome, obesity and diabetes. While in rodent model the link between fructose consumption and the cited metabolic perturbations is today clear, the evidence that this could take place in humans at the current level of fructose consumption remains under debate. What is the significance of the data described in animal models when translated to humans? Can we trust on fructose?
\end{abstract}

\section{Introduction}

Fructose is a natural sugar (with identical chemical formula than glucose) that can be found in small amounts in non-manufactured foods like fruits and honey. While fructose consumption has been traditionally low for humans (limited to the natural sources mentioned above) it has also increased dramatically in the last centuries, reaching $70 \mathrm{~kg} /$ person/year in 2006 [1]. To better understand this, we need to look back to the 1960s, when the industry of the US starts to produce in a very efficient and inexpensive way the so called high fructose corn syrup (HFCS). The HFCS is not more than just a mix (in different proportions) of corn-derived glucose and fructose, of which the most common is the $55 \%$ fructose $-42 \%$ glucose. As explained above, mainly due to its low costs of production, the HFCS has replaced other sugar sources in numerous food items, including soft drinks, sweet snacks and cereals.

\section{What is Wrong with Fructose Consumption?}

May be the first concern about fructose intake is its own metabolism. Unlike glucose, which is transported into hepatocytes through a GLUT2 carrier and metabolized through glycolysis up to the production of pyruvate and further acetyl-CoA, fructose does not follow the same pattern of degradation. Instead, fructose is absorbed in the gut (and partially metabolized in situ) through a GLUT5 carrier and then transported into the hepatocytes. Once inside, fructose is not metabolized through glycolysis, but converted into fructose-1phosphate and then into trioses phosphate, skipping to the control of insulin, ATP and citrate, and then being metabolized without further regulation [2]. The consequences are dramatic; since the liver became unable to handle the oxidation of the triose phosphate produced and then shifts the metabolism towards the glycogen synthesis and de novo lipogenesis, which ends by saturating the liver by lipids up to the development of a rapid steatosis.

The concern about the excessive fructose consumption started in the 1980s, when the HFCS starts to replace significantly other sugars in the food industry. Later, the consumption of the soft drinks containing high amounts of HFCS was suggested to increase in parallel with the obesity in the US and finally the large amount of animal studies pointed to the high fructose consumption as responsible for the development of several features of the metabolic syndrome in only a few weeks [3]. Among the metabolic perturbations induced by fructose intake at high proportions, the development of hypertriglyceridemia, glucose intolerance and insulin resistance are critical for the further installation of the metabolic syndrome. Other features also observed after the intake of large amounts of fructose also include the hypertension and a positive correlation with the acid uric levels.
The other reason why fructose consumption has attracted so much attention to the scientific community is because this sugar is not essential to any body function or metabolic functions in particular [4]. This is of special interest when comparing fructose to glucose, which is used for all cells of the human body as source of energy. In this sense, while the major carbohydrates dietary components consumed by man where traditionally those present in grains (most of them complex carbohydrates), fruit and honey, the total caloric sweeteners consumption in the USA and Europe constitutes about $20 \%$ of the total energy intake, with fructose representing $50 \%$ of this percentage [5].

\section{Have been the Effects Reported in Animal Models Observed in Humans?}

While today there is no doubt about the effects of large amounts of fructose intake (65\% of fructose) in rodent, when the impact of fructose consumption is discussed in the context of human metabolism and physiology, the results do not seem to be equivalent. In this sense, several critical reviews have discussed this matter in the last couple of years, putting in evidence the lack of sufficient data pointing to a deleterious fructose impact when consumed at moderate doses [4-7].

Most of the information relating the consumption of HFCS with obesity came from epidemiological studies. However, the direct evidence of such correlation is often limited [4]. Concerning short and long-term interventional studies in humans, the data is also under debate: at short-term Teff et al. [8], suggest that fructose leads to obesity by passing the insulin and leptin control. However, further studies demonstrated that the satiating effect of fructose drinks was not higher than other isoenergetic beverages [9] and that the consumption of sucrose-based drinks was not more beneficial than the consumption of those drinks containing single monosaccharides, like those present in the HFCS concentrates [10-12]. In the same sense, no studies up to date have demonstrated that fructose consumption could increase body weight at long-term in humans [13], at least with doses inferior at $100 \mathrm{~g} /$ day [14].

*Corresponding author: Sergio Polakof, INRA, UMR 1019, UNH, CRNH Auvergne, F-63000 Clermont- ferrand, France, E-mail : spolakofreq@clermont.inra.fr

Received August 20, 2012; Accepted August 22, 2012; Published August 24 2012

Citation: Polakof S, Sébedio JL, Comte B (2012) Sweet Dreams or Bitter Nightmare: Can We Trust on Fructose? Bioenergetics 1:e108. doi:10.4172/2167. $7662.1000 \mathrm{e} 108$

Copyright: (C) 2012 Polakof S, et al. This is an open-access article distributed under the terms of the Creative Commons Attribution License, which permits unrestricted use, distribution, and reproduction in any medium, provided the original author and source are credited. 
May be the aspect in which human studies follow the results reported using animal models is that related to the fructose-induced dyslipidemia [4,5]. Acute studies in humans confirm what we have learn from animal models, showing that fructose intake is able to increase the appearance of VLDL particles [15], postprandial lipogenesis [16] and circulating triglycerides levels [17]. Unfortunately, these interventions with human volunteers were carried out using fructose doses $(20-30 \%$ total energy intake) that largely exceed the current fructose consumption in Western countries (about 10\% total energy intake). What is then the relevance of such data? There is no doubt that fructose is able to alter significantly the lipid metabolism in humans, with deleterious effects at the short term. However, the doses used in the mentioned experiments are too high and then no conclusions can be drawn from moderate doses of fructose consumption. Given that no long-term studies have been performed so far, the real impact of moderate fructose consumption remains unknown. Moreover, while the increased triglycerides levels seem to be systematically demonstrated, even with high doses of fructose, the circulating concentrations remain far from those observed in diagnosed dyslipidemic patients [5]. Nevertheless, a possible deleterious effect of moderate fructose quantities cannot be discarded at the long-term, since high circulating triglycerides levels are associated with an increased risk of developing cardiovascular diseases, and it is worth mentioning that fructose has been already associated with hypertension in humans [18-20].

Another feature of the metabolic syndrome reported in rodent models feeding high fructose diet is the fast development of insulin resistance, which could be interpreted as a dysfunction preceding a diabetic state. Paradoxically, fructose has been traditionally considered as a performing therapy for diabetic patients given its low glycemic index and the inability to induce insulin secretion. Short-term studies in humans are, however, lacking and only after the intravenous infusion of fructose a whole body insulin resistance was observed [21]. Nevertheless, this study needs to be interpreted carefully, since does not evaluate the real effect of dietary fructose as inductor of insulin resistance. Chronic studies are more numerous, but no exempt of controversy. Effectively, fructose intake has been shown to induce insulin resistance in humans after 1 up to 10 weeks of consumption $[22,23,13]$. However, once again, the doses used are very high (about $3.5 \mathrm{~g}$ fructose/kg BW) often reaching 25\% of the total energy requirement for healthy humans, suggesting that such effects could not be interpreted for moderate doses. Actually, in those studies in which moderate doses of fructose were used no impact was observed on insulin sensitivity or glucose tolerance [5], suggesting that fructose is likely not responsible of the alterations observed in glucose homeostasis also in animal models. Whether or not longer exposures to fructose could, through secondary mechanisms, lead to insulin resistance remains to be elucidated.

\section{Finally: can we trust on fructose?}

The answer is that today there is not enough evidence of fructose having a deleterious effect on human health when consumed at moderate doses. It is, however, clear that numerous metabolic alterations do take place in high-fructose fed animal models, but most of them are no longer observed in humans with a normal fructose intake. Nevertheless, it is worth mentioning that given the increasing amount of fructose intake in the last 50 years in the Western countries and the evidence obtained from animal models, the excess of fructose in the diet should be conceived in the context of a healthier lifestyle, as the long-term effects of its consumption are still unknown.

\section{References}

1. Tappy L, Lê KA (2010) Metabolic effects of fructose and the worldwide increase in obesity. Physiol Rev 90: 23-46.

2. Le KA, Tappy L (2006) Metabolic effects of fructose. Curr Opin Clin Nutr Metab Care 9: 469-475

3. Basciano H, Federico L, Adeli K (2005) Fructose, insulin resistance, and metabolic dyslipidemia. Nutr Metab (Lond) 2: 5.

4. Rizkalla SW (2010) Health implications of fructose consumption: A review of recent data. Nutr Metab (Lond) 7: 82.

5. Tappy L, Le KA (2012) Does fructose consumption contributes to non-alcoholic fatty liver disease? Clinics and research in hepatology and gastroenterology.

6. Tappy L (2012) Q\&A: 'toxic' effects of sugar: should we be afraid of fructose? BMC Biol 10: 42

7. Collino M (2011) High dietary fructose intake: Sweet or bitter life? World J Diabetes 2: 77-81.

8. Teff KL, Elliott SS, Tschöp M, Kieffer TJ, Rader D, et al. (2004) Dietary fructose reduces circulating insulin and leptin, attenuates postprandial suppression of ghrelin, and increases triglycerides in women. J Clin Endocrinol Metab 89: 2963-2972.

9. Soenen S, Westerterp-Plantenga MS (2007) No differences in satiety or energy intake after high-fructose corn syrup, sucrose, or milk preloads. Am J Clin Nutr 86: 1586-1594.

10. Akhavan T, Anderson GH (2007) Effects of glucose-to-fructose ratios in solutions on subjective satiety, food intake, and satiety hormones in young men. Am J Clin Nutr 86: 1354-1363.

11. Stanhope KL, Griffen SC, Bair BR, Swarbrick MM, Keim NL, et al. (2008) Twenty-four-hour endocrine and metabolic profiles following consumption of high-fructose corn syrup-, sucrose-, fructose-, and glucose-sweetened beverages with meals. Am J Clin Nutr 87: 1194-1203.

12. Melanson KJ, Zukley L, Lowndes J, Nguyen V, Angelopoulos TJ, et al. (2007) Effects of high-fructose corn syrup and sucrose consumption on circulating glucose, insulin, leptin, and ghrelin and on appetite in normal-weight women. Nutrition 23: 103-112.

13. Stanhope KL, Schwarz JM, Keim NL, Griffen SC, Bremer AA, et al. (2009) Consuming fructose-sweetened, not glucose-sweetened, beverages increases visceral adiposity and lipids and decreases insulin sensitivity in overweight/ obese humans. J Clin Invest 119: 1322-1334.

14. Livesey G, Taylor R (2008) Fructose consumption and consequences for glycation, plasma triacylglycerol, and body weight: meta-analyses and metaregression models of intervention studies. Am J Clin Nutr 88: 1419-1437.

15. Chong MF, Fielding BA, Frayn KN (2007) Mechanisms for the acute effect of fructose on postprandial lipemia. Am J Clin Nutr 85: 1511-1520.

16. Parks EJ, Skokan LE, Timlin MT, Dingfelder CS (2008) Dietary sugars stimulate fatty acid synthesis in adults. J Nutr138: 1039-1046.

17. Teff KL, Grudziak J, Townsend RR, Dunn TN, Grant RW, et al. (2009) Endocrine and metabolic effects of consuming fructose- and glucose-sweetened beverages with meals in obese men and women: influence of insulin resistance on plasma triglyceride responses. J Clin Endocrinol Metab 94: 1562-1569.

18. Nguyen S, Choi HK, Lustig RH, Hsu CY (2009) Sugar-sweetened beverages, serum uric acid, and blood pressure in adolescents. J Pediatr 154: 807-813.

19. Jalal DI, Smits G, Johnson RJ, Chonchol M (2010) Increased fructose associates with elevated blood pressure. J Am Soc Nephrol 21: 1543-1549.

20. Perez-Pozo SE, Schold J, Nakagawa T, Sánchez-Lozada LG, Johnson RJ et al. (2010) Excessive fructose intake induces the features of metabolic syndrome in healthy adult men: role of uric acid in the hypertensive response. Int J Obes (Lond) 34: 454-461.

21. Dirlewanger M, Schneiter $P$, Jequier E, Tappy $L$ (2000) Effects of fructose on hepatic glucose metabolism in humans. Am J Physiol Endocrinol Metab 279 E907-E911. 
Citation: Polakof S, Sébedio JL, Comte B (2012) Sweet Dreams or Bitter Nightmare: Can We Trust on Fructose? Bioenergetics 1:e108. doi:10.4172/2167$7662.1000 \mathrm{e} 108$

Page 3 of 3

22. Beck-Nielsen H, Pedersen O, Lindskov HO (1980) Impaired cellular insulin binding and insulin sensitivity induced by high-fructose feeding in normal subjects. Am J Clin Nutr 33: 273-278.
23. Couchepin C, Lê KA, Bortolotti M, da Encarnaçao JA, Oboni JB, et al. (2008) Markedly blunted metabolic effects of fructose in healthy young female subjects compared with male subjects. Diabetes Care 31: 1254-1256. 